Craig, B. (1992). La femme face à la transmission des patrimoines au XIXe siècle: Droit, coutume et pratiques. Dans R. Bonnain, G. Bouchard \& J. Goy (éds)., Transmettre, hériter, succéder : la reproduction familiale en milieu rural FranceQuébec XVIIle-XXe siècles (pp. 231-241). Lyon: Presses universitaires de Lyon.

\section{LA FEMME FACE À LA TRANSMISSION DES PATRIMOINES AU XIXe SIĖCLE : DROIT, COUTUME ET PRATIQUES}

Béatrice Craig

Département d'histoire

Université d'Ottawa*

Jusqu'au début du XIXe siècle, la femme nord-américaine n'était pas considérée comme l'égale de l'homme, mais comme un être qui devait lui être subordonné. Le droit reflétait cet état d'esprit. La femme ne jouissait pas de droits civiques, et, si elle était mariée, ses droits à la propriété étaient tronqués.

Ces restrictions renforçaient l'autorité du père et mari à l'intérieur du ménage, l'épouse ne pouvant exercer qu'un contrôle très limité sur le patrimoine familial. Ce contrôle étendu du mari sur les biens de la famille renforçait aussi l'influence dont celui-ci pouvait jouir dans la communauté, à une époque où le statut socio-économique d'une famille reposait essentiellement sur la possession d'un capital et surtout d'un capital foncier.

La transmission des patrimoines jouant alors un plus grand rôle dans la reproduction sociale que le transfert d'un capital culturel ou social, les processus de transmission visaient d'abord à mettre entre les mains des garçons le capital initial indispensable au maintien de leur position dans la société dont ils faisaient partie.

Le développement du commerce et de l'industrie au XIXe siècle détendirent les liens entre capital, et surtout capital foncier, et statut social. Talent, éducation, diligence et entregent pouvaient, plus facilement que par le passé, compenser la faiblesse initiale du capital. Familles et société furent alors plus disposées à élargir le contrôle que les femmes en tant que filles, épouses ou veuves- pouvaient exercer sur le patrimoine familial, et même à le leur laisser exercer au détriment des hommes. L'évolution des idéologies au XIXe siècle facilita cette transformation. 
Aux Etats-Unis, les femmes se réclamèrent des principes énoncés dans la Déclaration d'Indépendance pour demander leur égalité devant le droit. A. partir des années 1840, les Etats du nord et de l'ouest réformèrent le droit à la propriété dans un sens plus favorable aux femmes. Le Canada angiais leur emboîta le pas un demi-siècle plus tard, l'industrialisation et les principes égalitaires progressant plus lentement de ce côté du $49 \mathrm{e}$ parallèle.

Qu'en fut-il au Canada français? Au Québec, l'opposition d'une église catholique très conservatrice et opposée à toute altération des relations intra-familiales repoussa les modifications du droit relatif aux patrimoines familiaux jusqu'au XXe siècle. Mais le droit peut être en retard sur les pratiques, et l'influence de l'Église avait des limites. La position des Canadiennes françaises vis-à-vis des patrimoines familiaux n'est pas un sujet qui a été beaucoup étudié. On peut certes glaner des informations sur la question dans la plupart des études existantes sur les systèmes de transmission des patrimoines, mais celles-ci ne sont pas rédigées du point de vue des femmes ; par conséquent, elles nous laissent une vision fragmenté, et surtout statique, de la question.

Les francophones du Haut-St-Jean offrent un territoire privilégié pour une étude empirique de l'impact d'un droit et d'une structure économique en évolution sur l'accès des femmes au patrimoine familial. Le Haut-St-Jean, aussi connu sous le nom de territoire du Madawaska, est situé à cheval sur la frontière canado-américaine, en partie dans le Maine et en partie au Nouveau-Brunswick.1. Ce territoire fit l'objet d'une querelle territoriale entre les Etats-Unis et la Grande-Bretagne jusqu'en 1842, date à laquelle le traité Webster-Ashburton fixa la frontière. La rive nord de la rivière resta britannique et sous la juridiction du N.B. La rive sud devint américaine, formant la partie septentrionale de l'état du Maine. Ce statut international incertain ne fit pas obstacle au peuplement. Le territoire fut ouvert à la colonisation par des Acadiens venus de la région de Fredericton, au N.B, en 1785. Dès le XVIle et jusqu'au XXe, il attira un flux relativement modeste, mais constant de Canadiens français en provenance du Bas-St-Laurent (surtout Kamouraska et Rivière Ouelle). La population anglophone du territoire ne représenta jamais plus de $10 \%$ de la population totale. En 1850, il y avait 6167 résidents dans le Haut-StJean, et près de 15000 en 1870 . La croissance démographique entraîna une occupation rapide des meilleures terres, en bordure du fleuve, qui furent toutes occupées dès les années 1840 . L'expansion territoriale subséquente requit le défrichement de sols plus pauvres. Le traité de 1842 stipulait que les colons authentiques recevraient des titres pour les terres qu'ils occupaient. Ce qui fut fait entre 1845 et 1848. Les Américains accordèrent des titres pour 563 lots; les Britanniques pour 505. Ensuite, les personnes désireuses de se procurer une terre étaient supposées l'acheter du gouvemement.

L'économie de cette région reposa presque exclusivement sur l'agriculture de subsistance jusqu'en 1825, une agriculture en voie de commercialisation et l'exploitation des ressources forestières ensuite. On commercialisation ex lexplaitation destance dans un peut présumer que le passage d'une économie d'auto-subsistance dans un territoire en expansion rapide, à une économie mixte, articulée sur des marchés extérieurs dans un territoire qui, s'il n'était pas saturé, était tout de même bien rempli, entraîna une attitude différente vis-à-vis du rôle de la terre dans l'économie familiale et dans le processus de reproduction sociale. Modifia-t-il les modes de transmission des patrimoines et l'accès des femmes à la propriété foncière?

Non seulement devons-nous nous demander si ces francophones furent assez sensibles à l'évolution des modes de production pour modifier leurs pratiques successorales, et l'accès des femmes à la propriété, mais nous devons aussi nous demander dans quelle mesure ils furent affectés par les transformations du droit. Ces habitants, largement originaires d'un pays de droit civil résidaient dans une contrée soumise à deux variantes de la Common Law anglo-saxonne. Or ces deux systèmes juridiques régissaient de manière très différente les rapports entre les membres de la famille et le patrimoine familial; de plus le Maine modifia le droit relatif aux biens des femmes mariés en 1846, accordant à ses citoyennes des droits que leurs consœurs du Canada durent attendre deux générations et plus.

Les francophones du Haut-St-Jean ont-ils alors cherché à préserver des pratiques importées du Bas-Canada, en dépit de l'évolution de l' économie et de leur sujétion à un droit à la fois étranger et lui aussi en évolution? Ont-ils cherché à préserver une conception traditionnelle des rapports entre maris et femmes? Ou combinèrent-ils des pratiques héritées de leurs aïeux avec des éléments de la Common Law pour mieux s'adapter aux conditions économiques nouvelles? Quelles furent leurs stratégies patrimoniales pour sauvegarder leurs intérêts dans ce contexte mouvant? Et comment les femmes en furent-elles affectées? 


\section{I - LA TRANSMISSION DES PATRIMOINES DANS LE NORD-EST AMÉRICAIN}

\section{A. Le droit}

Sujets britanniques ou citoyens américains, les francophones du Haut-St-Jean furent assujettis à un droit qui leur était étranger : la Common Law. L'attitude vis-à-vis des relations intra-familiales et du contrôle des biens du ménage qui sous-tend la Common Law d'une part, la coutume de Paris, en vigueur au Bas-Canada jusque 1866, et le Code civil qui lui succéda de l'autre sont antithétiques ${ }^{2}$.

Le droit français relatif aux patrimoines familiaux s'articulait autour du concept de la communauté des biens, qui était le régime matrimonial normal au Bas-Canada. Le concept de communauté était étranger a la Common $L a w$, et la séparation de biens était l'exception et non la règle. Jusqu'au début du XIXe siècle, la Common Law traitait les biens meubles possédés par le mari ou la femme avant le mariage, ainsi que tous les biens acquis depuis, y compris les meubles reçus en héritage, comme étant la propriété du mari. Le mari de plus, administrait les biens fonciers que sa femme possédait au moment du mariage. Au décès du mari, la veuve se voyait attribuer un douaire, sorte de pension alimentaire consistant en un tiers des meubles de son mari et l'usufruit d'un tiers de ses immeubles. Le veuf par contre se voyait accorder l'usufruit des biens fonciers que sa femme pouvait posséder s'ils avaient eu au moins un enfant vivant. Les femmes pouvaient protéger leurs intérêts matériels en concluant un contrat de mariage, mais ceux-ci étaient fort rares. A partir de 1840 , un nombre croissant d'Etats américains promulga des lois relatives aux biens des femmes mariées, qui permettaient à celles-ci d'administrer et de disposer à leur gré des biens qu'elles avaient apporté en mariage ou reçu depuis en héritage. Une génération s'écoula avant que ces lois ne s'étendent au fruit de leur travail. Les provinces canadiennes anglaises furent beaucoup plus lentes à reconnaître aux femmes mariées des droits à la propriété ; elles ne promulguèrent de lois semblables que dans les années 1880 et 1890 .

En cas de succession intestat, le droit français et le droit angloaméricain exigeaient l'égalité des héritiers. La primogéniture par contre était la règle dans l'Amérique du Nord britannique en l'absence de testament; elle fut abolie au Nouveau-Brunswick en 1854. Le droit anglais accordait au père pleine liberté testamentaire: il pouvait modifier à volonté les prescriptions du droit. Le droit français limitait très sévèrement la liberté testamentaire; celle-ci fut introduite au Canada français par l'Acte de Québec en 1774, mais cette clause semble n'avoir eu aucun impact sur les pratiques avant la fin du XIXe siècle ${ }^{3}$. Toutes ces sociétés cherchaient normalement à donner aux fils leur part en terres, et aux filles en biens meubles; dans certaines colonies américaines, la loi avait d'ailleurs exigé que ce soit le cas, si cela était possible.

La philosophie qui sous-tend les deux droits reflète deux attitudes différentes par rapport aux relations familiales. Le droit anglais était plus patriarcal ; il donnait au mari un plus grand contrôle sur sa femme et ses enfants, même après sa mort. II considérait la femme, avant ou après son veuvage, comme économiquement dépendante de son mari, et ne tenait pas compte de sa participation à l'économie familiale. Le droit français, par contre, reconnaissait la participation économique des femmes à l'enrichissement du ménage, et considérait que cette participation était égale à celle du mari ; il donnait aux veuves une chance d'accéder à l'indépendance économique.

\section{B. Les pratiques}

Les normes et les pratiques ne sont toutefois pas toujours identiques. L'historiographie canadienne française, tout comme certaines études sur le nord-est américain (et même sur le Haut-Canada), suggère que les pratiques de ces sociétés se ressemblaient beaucoup plus que le droit ne le 1aisse croire. Chaque génération de familles de fermiers, dans chacune de ces sociétés, était confrontée au même dilemme: comment établir de nombreux enfants, à partir des ressources d'une seule exploitation, sans menacer l'intégrité économique du patrimoine ou la sécurité des parents âgés.

Les stratégies décrites par les historiens canadiens et américains sont similaires ${ }^{4}$. Les parents s'efforçaient d'acquérir des parcelles supplémentaires, par achat ou défrichement, et les transféraient à leurs fils quand ceux-ci se mariaient. S'il n'existait pas localement de terres disponibles à un prix abordable, les parents avaient recours à d'autres stratégies pour établir leurs fils. S'ils résidaient dans une région anciennement peuplée, ils pouvaient vendre leur ferme à bon prix, et utiliser cet argent pour acquérir plusieurs lots sur un front de colonisation, où la terre était meilleur marché.

Au Canada français, le père âgé transmettait sa demière parcelle (ou sa seule parcelle s'il n'en avait qu'une) à un fils, qui était tenu de subvenir aux besoins de ses parents pendant leur vieillesse, mais aussi de dédommager ses frères et sœurs. Ces demiers pouvaient employer le 
capital ainsi reçu pour aller s'établir dans une zone de colonisation. On ne donnait pas habituellement de terres aux filles, mais elles n'étaient pas exclues de la distribution du patrimoine familial : elles recevaient une dot en meubles, bétail, linge ou argent au moment de leur mariage ou à la mort de leurs parents. Les fermiers américains soit avaient recours à une donation, soit transféraient la terre à un fils, mais s'en réservaient l'usage pour le reste de leurs jours (et imposaient à leurs fils le paiement d'une pension alimentaire à la veuve).

Les conséquences des deux systèmes pour les veuves étaient semblables. Les veuves américaines avaient droit à un douaire, une pension alimentaire prise sur la succession de leur mari ; cette pratique sacrifiait leur indépendance financière à celle de leurs enfants. Les veuves canadiennes françaises avaient droit à la moitié des biens de la communauté, mais si celle-ci avait été distribuée aux enfants au fur et à mesure de leur mariage, elles ne pouvaient compter, elles aussi, que sur une pension après le décès de leur mari.

Les systèmes de dévolution nord-américains visaient donc à mettre la terre entre les mains des fils, aux dépens des filles et des veuves, dont les droits au patrimoine familial étaient limités. Les filles recevaient les biens requis pour assumer leurs responsabilités au sein de leurs ménages: meubles meublants, animaux, quelquefois de l'argent. Les veuves se voyaient accorder une pension en nature ou en espece. Ces systèmes toutefois prévalaient dans des sociétés où l'agriculture était peu commercialisée, et où les possibilités d'emploi étaient limitées. L'apparition d'une agriculture commerciale et l'expansion du marché de l'emploi altéraient profondément les stratégies successorales. Selon Toby Ditz, la commercialisation de l'agriculture au Connecticut dans les années 1820 entraina l'adoption de deux stratégies successorales diamétralement opposées : ou bien la ferme était transmise, intacte, à un seul héritier, qui n'était plus tenu de compenser ses frères et sœurs (une évolution relevée par Greer dans le Richelieu à la même époque) ; ou bien la terre était traitée comme un bien meuble, partagée ou vendue par les héritiers. Lorque la terre était assimilée à un bien meuble, les filles étaient incluses dans la distribution, et les veuves se voyaient reconnaître des droits accrus envers le patrimoine familial, même aux dépens des enfants. Comme le dit Ditz: «When productive property ceases to be important, women's status with respect to control over property improves ${ }^{3}$.
II - LA TRANSMISSION DES PATRIMOINES DANS LE HAUT-ST-JEAN

Cette étude s'appuie sur le relevé intégral des actes enregistrés entre 1845 et 1870 aux bureaux d'enregistrement des terres dont dépendait le Haut-St-Jean. Les différents actes ont été regroupés sur des fiches-terriers au nom des personnes impliquées; ces fiches ont ensuite été jumelées avec les fiches de reconstitution des familles qui avaient été établies pour une étude antérieure. Il y eut 3197 transactions enregistrées entre 1845 et 1871 . Dans $5,7 \%$ des cas [ $N=182$ ], l'une des personnes engagées dans la transaction était une femme. Le pourcentage est plus élevé du côté américain $(6,4 \%)$ que canadien $(4,6 \%)$, et implique surtout $70 \%[N=88]$ des femmes mariées. Le marché foncier reste donc un marché d'hommes. Les femmes y apparaissent dans des circonstances très particulières. L'ensemble des transactions révèle toutefois une attitude envers la propriété foncière qui se démarque de celle qui prévalait dans les régions d'agriculture de subsistance. Dans le Haut-St-Jean, le couple était au centre de la vie sociale et économique. Les enfants avaient droit au gîte et au couvert jusqu'à leur mariage, à une modeste dot ensuite. Les parents étaient plus préoccupés par la sécurité matérielle de leur vieillesse que par l'établissement de leurs enfants sur une terre. L'indépendance des veuves n'était pas communément subordonnée aux besoins des enfants; et les résidents de la rive américaine intégrèrent le Married Women's Property Act dans leurs stratégies foncières.

Quoique les transferts inter vivos aient été la norme ( $2 / 3$ des couples aliénaient toutes leurs terres de leur vivant), cette société n'accumulait pas les terres pour établir ses fils au moment de leur mariage : seule la moitié des familles possédait plus d'un lot ${ }^{6}$. Le transfert de terres de parents à enfants ne coüncidait pas avec le mariage de ces demiers, et prenait presque toujours la forme soit d'une vente, pour ce qui semble avoir été le prix du marché, soit d'une donation contre rente viagère. La majorité des transferts à enfants étaient des transferts à des fils, le reste, à des gendres. Les filles étaient donc normalement exclues de ce type de partage en tant qu'individus, mais leurs familles ne l'etaient pas.

Les obligations du récipiendaire d'une terre contre rente viagère se limitaient à celles envers le couple donateur, et envers les enfants vivant encore sous le toit familial à ce moment là. Ces demiers avaient droit au gîte et au couvert jusqu'à leur majorité ou leur mariage, à condition de travailler pour leur frère. Quand un jeune se mariait ou quittait la ferme familiale, il ou elle avait droit à un trousseau. Les filles recevaient toujours une vache, et parfois un lit avec literie, un rouet, des brebis. Les 
garçons avaient droit à un cheval de trois ans, et quelquefois des meubles.

Le patrimoine foncier n'était pas non plus réservé aux enfants. La moitié des couples considérés aliéna une partie de ses terres hors de la famille immédiate. Un peu moins d'un tiers ne transféra des biens fonciers qu'à des enfants; une proportion similaire exclut les enfants de tous leurs transferts. Dans ces demiers cas, il ne s'agissait pas de ventes destinées à financer le ré-établissement de toute la famille sur un front pionnier où il serait possible d'acquérir une abondance de terre à bas prix. Les couples mariés dans le Haut-St-Jean avant 1850 en effet n'émigraient pas. Les couples qui accumulaient des parcelles supplémentaires pendant leur mariage ne se comportaient pas de manière différente : en fait, plus un couple possédait de terres, plus il était susceptible d'en aliéner au moins une partie hors de la famille immédiate.

Filles et garçons étaient donc mis sur un pied de quasi-égalité : les garçons ne recevaient pas plus à titre gratuit que leurs sœurs, et ne pouvaient prétendre qu'à une préférence lorsque leurs parents décidaient de vendre ou de donner leur terre en viager. Les parents n'accumulaient pas pour le bénéfice de leur descendance, mais pour le leur propre.

Les parents semblent avoir cherché a atteindre deux buts successifs : d'abord, élever leur nombreuse famille (une moyenne de 11 enfants par famille complète avant 1850), et modestement doter les enfants, et ensuite se garantir un revenu suffisant pour leur vieillesse. Le destin des veuves qui avaient des enfants mineurs, et que l'on peut suivre dans les sources renforce cette impression.

Lorsque la mort prenait une famille par surprise, surtout si le mari était encore jeune, la norme semble avoir été de ne pas régler la succession avant que le benjamin n'atteigne sa majorité. A ce moment-là la veuve pouvait racheter les parts de ses enfants, et échanger l'exploitation contre une pension. Il était aussi fréquent que la veuve reprenne l'exploitation pour élever les enfants, puis l'utilise pour couvrir ses besoins pendant sa vieillesse. Par exemple, en 1860, Louise Albert, une veuve de 58 ans, racheta aux héritiers les neufs concessions laissées par son mari pour la somme totale - et relativement insignifiante - de $£ 50$ (\$200.). Ensuite, elle les revendit à ses fils à des prix beaucoup plus proches du marché. Dans un cas de décès prématuré, la jeune veuve vendit, pour la somme de $\$ 250$, son douaire dans les biens nombreux, mais très hypothéqués, de son mari, ce qui lui permit de s'acheter une terre, certes modeste, mais libre de dettes.
Les hommes qui désiraient garantir l'indépendance économique de leur veuve, tout en gardant le controle de leurs terres leur vie durant, pouvaient rédiger un testament. Peu se prévalurent de cette option: je n'ai retrouvé, dans les tribunaux d'homologation, et dans les registres des terres, que 23 testaments pour la période 1845--1870. Les hommes jeunes n'envisageaient pas la possibilité de leur décès prématuré ; les hommes âgés assuraient la sécurité de leur veuve, comme la leur, par le biais de la donation contre rente viagère. Dans leur ensemble, les testaments étaient plus favorables aux veuves que le droit anglais ou français. Dans $40 \%$ des cas, la veuve recevait au moins la moitie des biens meubles et immeubles en pleine propriété, et dans $17 \%$ des cas, elle recevair l'usufruit de la totalité du patrimoine familial. La plupart des hommes qui laissaient un testament accordaient la priorité aux besoins de la veuve, et non à ceux des enfants. Souvent, ils ne désiraient pas que leurs biens soient partagés avant la mort de leur épouse. Le testament de Jean-Baptiste Daigle III illustre bien la tendance précédente. Il laissa à sa femme une section de 20 perches de large de la ferme familiale, avec la maison, l'étable, le mobilier, le bétail et le matériel agricole. Le reste de ses terres, 10 perches de la ferme familiale et un lot de 100 perches de large à defricher devait être partagé entre les garçons. Les filles devaient recevoir $\$ 25$ chacune à leur majorité. Les biens laissés à la veuve valaient probablement plus que la moitié de la succession ; de surcroît, elle devait jouir de l'usufruit du reste pour élever les 9 enfants, âgés de 2 à 16 ans au décès du père.

La loi sur les biens des femmes mariées permit aussi aux hommes qui possédaient des terres du côté américain de mettre des biens fonciers entre les mains de leur femme. Quoiqu'on y eut recours dès son passage, peu s'en prévalurent ( 24 pour la période 1846-1870). Le petit nombre ne permet pas de dégager un portrait-robot de ces maris, mais l'ensemble des cas relevés suggère qu'on se prévalait de cette loi pour faire face à des situations qui s'écartaient de la norme ; remariage avec une femme plus jeune, mésentente conjugale, dans les cas où le mari mettait une partie de ses biens au nom de sa femme a condition de ne plus avoir a subvenir a ses besoins, mauvaise gestion par le mari quand ce dernier transférait ses biens à son épouse qui en retour devait rembourser ses dettes. A l'exception des cas de séparation, le but de ces transferts n'était pas d'assurer l'indépendance économique de l'épouse, mais de renforcer la sécurité matérielle des ménages puis, en cas de décès du mari, l'indépendance de la veuve. Plus encore que le testament, la donation garantissait l'indépendance de celle-ci, libre de toute ingérence des enfants du premier ma- 
riage. Par contre, une donation permettait à la femme de disposer comme elle l'entendait de la terre, même du vivant du mari. Ces demiers, de toute évidence, n'étaient pas d'humeur patriarcale.

Les maris ne semblaient d'ailleurs pas très intéressés à contrôler leurs épouses après leur mort. Un seul mari, par exemple, stipula que les clauses de son testament, avantageuses pour sa veuve, seraient invalidées si elle se remariait. Ce refus de contrôler épouses et héritiers par delà la tombe influença même une famille locale d'anglophones. En 1867, John Emmerson, le plus riche habitant permanent du Haut-St-Jean, et marchand de profession mourut, laissant un testament tâtillon qui n'accordait aucune autonomie à sa veuve. Mais ses fils s'empressèrent de mettre au nom de leur mère les biens dont ils héritèrent - ce qui était tout à fait légal, mais non dans l'esprit du testament. Sept ans plus tard, Jane Emmerson rédigea à son tour son testament. Elle légua ses biens fonciers à ses fils, et laissa à sa seule fille, mariée à un marchand du Bas-Canada, 20 actions de la Banque Nationale de Québec «en son nom à elle, et libre de toute ingérence de la part de son mari». Par conséquent un testament qui violait les normes communautaires pouvait ne pas être respecté.

Les habitants du Haut-St-Jean nous frappent par leur souplesse, leur pragmatisme et leur ingéniosité. Ils tiraient parti de toutes les opportunités possibles pour mettre sur pied des stratégies visant, non pas à établir le plus de fils possible sur des terres, mais à assurer la sécurité matérielle du couple ou de son survivant, et à le mettre en mesure d'élever les enfants jusqu'à leur majorité. Ce désintérêt pour le sort des enfants adultes était possible, parce que ceux-ci pouvaient s'établir sans l'aide des parents : l'existence de terres défrichables, d'emplois dans les chantiers permettaient à ceux qui restaient d'assurer leur subsistance. Si celle-ci paraissait trop modeste, l'émigration était une option réaliste : le St-Jean traversait l'une des routes migratoires du Bas-Canada vers les centres industriels de Nouvelle-Angleterre, et les habitants de la vallée prirent le chemin «des Etats» dès les années 1840. Ils s'en allèrent chercher fortune jusqu'au Montana. Non seulement l'agriculture locale se commercialisait, mais le St-Jean s'articula immédiatement aux marchés du travail qui s'ouvraient dans d'autres Etats. Et comme dans le Connecticut deux générations plus tôt, lorsqu'il ne fut plus nécessaire de trouver une terre pour les fils pour assurer leur statut social, les femmes se virent reconnaittre des droits accrus au patrimoine familial.

\section{NOTES}

* Ce travail a bénéficié d'une subvention đu Centre de recherche en civilisation canadienne-français de l'Université d'Ottawa.

1. Pour plus d'informations sur cette région, yoir : B. Craig, «Migrant Integration and Kinship Ties in Frontier Community, Madawaska 1986, p. 277-297: id., *Agriculture and the Lumberman's Frontier in the Upper St John Valley, 1800-1870», Journal of Forest History, vol. 32, July 1988, p. 125-137.

2. La discussion qui suit s'appuie sur C. Shammas, M.Salmon and M. Dahlin, Inheritance in America, from Colonial Times to the Present, New Brunswick, New Jersey, Rutgers University Press. 1987 : J. Boucher, kL'histoire de la condition juridique et sociale de la femme au Canada français». dans J. Boucher et A. Morel, Le Droit dans la vie familiale, vol. 1. Montréal, Presses de l'Universite de Montreal, 1970: S. Altschol and C. Carron, «Chronology of Some Legal Landmarks in the History of Canadian Womenn, McGill Law Journal, vol. 21, 1975. p. 476-494: R. Chused, «Married Women's Propenty Law. 1800-1850», Georgetown Law Journal, vol. 71, 1983, p. 1359-1425.

3. A. Greer. Peasant, Lord and Merchant, Rural Society in Three Quebec Parishes, 1740-1840. Toronto. University of Toronto Press, p.81, note 77.

4. Les sources sur lesquelles s'appuie cette description sont trop nombreuses pour être citées in extenso. Pami les principales, citons: G. Bouchard, "Les systèmes de transmission des avoirs familiaux et le cycle de la societé rurale au Québec du XVile au XXe *. Histoire sociale - Social History,
vol. XVI, mai-May 1983, p. 35-60.: id., * Sur la reproduction familiale en milieu rural : systemes vol. XVI, mai-May 1983, p. 35-60.; id., "Sur la reproduction familiale en milieu rural : systemes ouverts et systèmes closm, Recherches sociographiques, vol. XVIII. 1987, p. 229-251; Louise Dechêne, Habitants el marchands de Montreal. Paris, Plon, 1979, p. 418-424; A. Greer. op.cit,
T. Ditz Property and Kinship. Inheritance in Early Connecticut, 1750-1820. Princeton University T. Ditz, Property and Kinship, Inheritance in Early Connecticut, Inso-1820. Princeton University
Press, $1986 . \mathrm{J}$. Henretta, «Families and Farms, Mentalité in Pre-Industrial America». William and Press, 1986 : J. Henretta. «Families and Farms, Mentolite in Pre-Industral America», Whiliam and Mary Quarterly, 3d, ser.. vol. XXXV, 1978, p. 3-33; J.J. Waters, kFamily, Inheritance and Migration
in Colonial New England: The Evidence from Guildford, Connecticuts, William and Mary Quarterly, 3d. ser., vol. XXXXIX, January 1982, p. 64-86; voir aussi la bibliographie dans : B Quarerly. Jd. ser., vol. XXXXX, January 1'étude des sociétés rumes nord-américaines», Hisioire sociale-Social History, vol. XXIII. novembre 1990, p. 249-270.

5. T. Ditz, Property and Kinship. op.cit.. p. 36.

6. Les conclusions qui suivent reposent sur l'analyse de deux sous-populations. La première est constituée par les familles dont le chef est décédé entre 1845 et 1870 [ $N=180]$ : la seconde par celles dont le chef est né entre 1785 et 1805 [ $\mathrm{N}=306]$. Pour une analyse plus détaillée des systèmes de transmission des patrimoines dans cette région voir: B. Craig. "La transmission des patrimoines fonciers dans le paut St Jean a XIXe siecle» communication presenté au Congres de la Sociéte

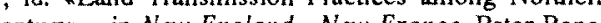
Maine French Canadians in the Nineteenth Century», in New England - New France, Peter Benes 